\title{
Persistent Spin Currents in Helimagnets
}

\author{
Jan Heurich, ${ }^{1}$ Jürgen König, ${ }^{1}$ and A. H. MacDonald ${ }^{2}$ \\ ${ }^{1}$ Institut für Theoretische Festkörperphysik, Universität Karlsruhe, 76128 Karlsruhe, Germany \\ ${ }^{2}$ Department of Physics, University of Texas at Austin, Austin, TX 78712
}

(Dated: November 15, 2018)

\begin{abstract}
We demonstrate that weak external magnetic fields generate dissipationless spin currents in the ground state of systems with spiral magnetic order. Our conclusions are based on phenomenological considerations and on microscopic mean-field theory calculations for an illustrative toy model. We speculate on possible applications of this effect in spintronic devices.
\end{abstract}

PACS numbers: 75.10.Lp,72.25.Ba,74.20.-z,72.15.Nj

\section{INTRODUCTION}

Collective transport effects in ordered many-fermion and many-boson systems include some of the most dramatic and profound phenomena that occur in condensed matter physics. For example, the (practically) dissipationless transport of electrical charge by Cooper pairs 1 in superconductors and superfluidity in ${ }^{4} \mathrm{He}$ and ${ }^{3} \mathrm{He}$ have been important topics through most of the field's history. New instances of this general class of phenomenon continue to arise and create interest. One recent case is collective charge transport in double-layer quantum Hall systems, in which spontaneous inter-layer phase coherence leads to a strongly enhanced zero-bias tunnel current from one layer to the other 2.3 .4 .5 Closely related issues connected with the possibility of superfluidity due to excitonic Bose condensation in electron-hole doublelayer systems $\frac{6.7}{}$ also continue to attract attention ${ }^{\underline{8}}$ and inspire experimental activity.

Two of us $\frac{9}{}$ have recently proposed the possibility of realizing nearly dissipationless collective spin currents in easy-plane thin-film ferromagnets. Although the proposed phenomenon has some formal relationship to superconductivity and superfluidity, it depends in part on a symmetry (magnetization orientation invariance within an easy plane) that can only be realized approximately. More importantly, unlike the case of superconductors which can easily be biased by a current source, strategies for driving a real thin-film ferromagnet into the metastable spin-current state present experimental and materials challenges that have not yet been overcome. For ferromagnets, the meta-stable states that have a nonzero spin current are spiral states, as sketched in Fig. [1

In Ref. 9 we demonstrated that these states are

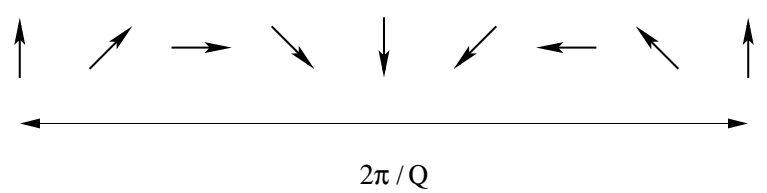

FIG. 1: State with spiral magnetic order in the $\hat{\mathbf{x}}-\hat{\mathbf{y}}$ plane characterized by the wave vector $Q$. The arrows represent the local spin density of the itinerant-electron system. metastable only when the system has a non-zero magnetic anisotropy that is accurately uniaxial with an easy plane, and proposed an experimental strategy to generate and detect macroscopic dissipationless spin currents in thin-film ferromagnets. Other related recent work has addressed persistent spin currents in rings that experience an inhomogeneous magnetic field ${ }^{10.11 .12}$ In this paper we consider the case in which the ground state of the system has spiral order, the case of helimagnets. As mentioned already in Ref. 9, the persistent spin current of a spiral state is proportional to the derivative of its energy with respect to the state's winding wavevector $\mathbf{Q}$, a quantity which necessarily vanishes in the ground state. Like ferromagnets, helimagnets do not normally carry a spin current in their ground state. However, as we demonstrate in this paper, in the helimagnet case a persistent ground state spin current is induced by an external magnetic field.

Throughout this paper we consider states in which the spin density $\langle\mathbf{s}(\mathbf{r})\rangle$ lies in the $\hat{\mathbf{x}}-\hat{\mathbf{y}}$ plane. The stability of these states will, in general, require some easyplane anisotropy that could have magnetostatic or magnetocrystalline origin. The presence of such a magnetic anisotropy energy term is implicitly assumed throughout the paper. In the first part of the paper, we set the applied magnetic field to zero and consider states with simple spiral order, illustrated schematically in Fig. 1] Our objective in this section is to demonstrate by an explicit microscopic calculation the property that the spiral ground states of helimagnets, unlike the spiral metastable excited states of ferromagnets, do not carry persistent spin currents. The order parameter of a spiral state is

$$
\langle\mathbf{s}(\mathbf{r})\rangle_{\mathbf{Q}}=s_{\mathbf{Q}}[\cos (\mathbf{Q} \cdot \mathbf{r}) \hat{\mathbf{x}} \mp \sin (\mathbf{Q} \cdot \mathbf{r}) \hat{\mathbf{y}}] .
$$

and it is characterized by a wave vector $\mathbf{Q}$, by the magnitude $s_{\mathbf{Q}}$ of the order parameter, and by a chirality. For a single-band lattice model $0 \leq s_{\mathbf{Q}} \leq 0.5 n_{\mathrm{e}}$, where $n_{\mathrm{e}}$ is the electron density per atomic site. As $\mathbf{Q}$ is varied, the change of the order parameter's spatial dependence will cause a change in the magnetic condensation energy and in the magnitude of the order parameter. We have argued previously ${ }^{9}$ that these systems carry a dissipationless spin current, i.e., a current with equal magnitude and opposite directions for up and down spins, that is 
related to the dependence of the total energy density $\epsilon$ on $\mathbf{Q}$ by $^{9}$,

$$
\mathbf{j}_{\uparrow}=\frac{e}{\hbar} \frac{\partial \epsilon(\mathbf{Q})}{\partial \mathbf{Q}}=-\mathbf{j}_{\downarrow}
$$

Note that the spin quantization axis is along the $\hat{\mathbf{z}}$ direction here. All spin currents discussed in this paper are polarized along the direction perpendicular to the plane defined by the spiral order parameter; we therefore do not explicitly indicate the tensor character of spin currents. Eq. (1.2) implies that it is not the presence of spiral magnetic configurations, but rather the change of total energy with varying spiral wave vector $\mathbf{Q}$ which leads to spin currents 13 . If the energy has a local minimum at a finite wave vector $\mathbf{Q}^{\star}$ (as in a helimagnet) then this state will, unlike spiral states in a system with a ferromagnetic ground state, not support a persistent spin current. We examine this property from a microscopic point of view in Section II. In Section III we discuss persistent spin currents using the picture of "Cooper pairs" familiar from the Bardeen-Cooper-Schrieffer (BCS) mean-field theory of superconductivity, which provides an alternative way of understanding when and why spin supercurrents appear for a given magnetization configuration.

With this background, we turn our attention in Section IV to a new strategy for generating persistent spin currents. We point out that persistent spin currents can be generated in systems with spiral magnetic order simply by applying a magnetic field oriented in the plane of spiral order, the $\hat{\mathbf{x}}-\hat{\mathbf{y}}$ plane in our notation. The external magnetic field creates a competition between spiral order with wave vector $\mathbf{Q}^{\star}$ and homogeneous magnetization along the field direction, leading to magnetic order described by a soliton lattice with wave vector $|\mathbf{Q}|<\left|\mathbf{Q}^{\star}\right|$. We demonstrate that soliton-lattice states carry persistent spin currents, whose amplitude is controlled by the strength of the applied magnetic field. Finally, we conclude in Section $\mathrm{V}$ with some brief speculations on possible applications in spintronics for these field-induced spin currents.

\section{MICROSCOPIC MODEL FOR PERSISTENT SPIN CURRENTS IN HELIMAGNETS}

\section{A. Hartree-Fock theory for spiral magnetic order}

In this section we try to shed additional light on the relationship between persistent spin currents and the dependence of energy on the spiral wavevector by carrying out explicit calculations for a simple toy model of an itinerant-electron system with magnetic order. Similar spiral state models have been presented previously ${ }^{14,15}$; the following brief description is included for completeness and intended to establish notation for the following discussion.

We consider a system of fermions on a lattice with single-particle band energy $\epsilon_{\mathbf{k}}$ and delta-function repulsive particle-particle interaction $U \delta\left(\mathbf{r}_{i}-\mathbf{r}_{j}\right)$, which we treat in a mean-field approximation. The unrestricted Hartree-Fock mean-field Hamiltonian for the spiral ordered state with wave vector $\mathbf{Q}$ is

$$
\mathcal{H}^{\mathrm{HF}}=\frac{V h^{2}}{U}+\sum_{\mathbf{k}}\left(c_{\mathbf{k}+\mathbf{Q} / 2, \uparrow}^{\dagger} c_{\mathbf{k}-\mathbf{Q} / 2, \downarrow}^{\dagger}\right)\left(\begin{array}{cc}
\epsilon_{\mathbf{k}+\mathbf{Q} / 2} & -h \\
-h & \epsilon_{\mathbf{k}-\mathbf{Q} / 2}
\end{array}\right)\left(\begin{array}{c}
c_{\mathbf{k}+\mathbf{Q} / 2, \uparrow} \\
c_{\mathbf{k}-\mathbf{Q} / 2, \downarrow}
\end{array}\right)
$$

where $h=U s_{\mathbf{Q}}$ and $s_{\mathbf{Q}}=(1 / V) \sum_{\mathbf{k}}\left\langle c_{\mathbf{k}+\mathbf{Q} / 2, \uparrow}^{\dagger} c_{\mathbf{k}-\mathbf{Q} / 2, \downarrow}\right\rangle$. The off-diagonal terms in Eq. (2.1) couple electronic states with opposite spin and Bloch wave-vector difference Q. (The wavevectors above are understood to be reduced to the first Brillouin-zone of the lattice.) To diagonalize the Hamiltonian we employ the transformation

$$
\left(\begin{array}{c}
a_{\mathbf{k},+} \\
a_{\mathbf{k},-}
\end{array}\right)=\left(\begin{array}{cc}
\cos \Theta_{\mathbf{k}} & -\sin \Theta_{\mathbf{k}} \\
\sin \Theta_{\mathbf{k}} & \cos \Theta_{\mathbf{k}}
\end{array}\right)\left(\begin{array}{c}
c_{\mathbf{k}+\mathbf{Q} / 2, \uparrow} \\
c_{\mathbf{k}-\mathbf{Q} / 2, \downarrow}
\end{array}\right)
$$

with $\tan 2 \Theta_{\mathbf{k}}=2 h /\left[\epsilon_{\mathbf{k}+\mathbf{Q} / 2}-\epsilon_{\mathbf{k}-\mathbf{Q} / 2}\right]$ and $0 \leq \Theta_{\mathbf{k}}<\pi / 2$ to arrive at $\mathcal{H}^{\mathrm{HF}}=\left(V h^{2} / U\right)+\sum_{\mathbf{k}, \pm} E_{\mathbf{k}}^{ \pm} a_{\mathbf{k}, \pm}^{\dagger} a_{\mathbf{k}, \pm}$. The eigenenergies of the ordered-state quasiparticles are given (for $h \geq 0$ ) by

$E_{\mathbf{k}}^{ \pm}=\frac{\epsilon_{\mathbf{k}+\mathbf{Q} / 2}+\epsilon_{\mathbf{k}-\mathbf{Q} / 2} \pm \sqrt{\left(\epsilon_{\mathbf{k}+\mathbf{Q} / 2}-\epsilon_{\mathbf{k}-\mathbf{Q} / 2)^{2}+4 h^{2}}\right.}}{2}$
The effective magnetic field which helps split the quasiparticle bands is fixed by solving the self-consistency equation $h=U s_{\mathbf{Q}}$. For this simple model an explicit expression can be given for the right hand side and we obtain

$$
\frac{U}{V} \sum_{\mathbf{k}} \frac{f\left(E_{\mathbf{k}}^{-}\right)-f\left(E_{\mathbf{k}}^{+}\right)}{\sqrt{\left(\epsilon_{\mathbf{k}+\mathbf{Q} / 2}-\epsilon_{\mathbf{k}-\mathbf{Q} / 2)^{2}+4 h^{2}}\right.}}=1
$$

where $f(E)$ is the zero-temperature Fermi function with the chemical potential determined by $(1 / V) \sum_{\mathbf{k}}\left[f\left(E_{\mathbf{k}}^{-}\right)+\right.$ $\left.f\left(E_{\mathbf{k}}^{+}\right)\right]=n_{\mathrm{e}}$.

\section{B. Specific toy model}

The above equations are valid for an arbitrary band dispersion relation $\epsilon_{\mathbf{k}}$. In Ref. 9 free fermions with 
parabolic dispersion were considered. For this case states with spiral magnetic order always have higher energy than uniform magnetization (ferromagnetic) states. In the present paper, however, we want to construct a model in which the minimum (mean-field theory) energy occurs for a spiral state with wave vector $\mathbf{Q}^{\star} \neq 0$, i.e., a model for which spiral magnetic order is favored over ferromagnetism ${ }^{16}$ This circumstance is achieved most simply by choosing a quasi-one dimensional model, i.e., by choosing

$$
\epsilon_{\mathbf{k}}=\frac{W}{2}\left[1-\cos \left(k_{z} a\right)\right]
$$

for $-\pi / a \leq k_{z} \leq \pi / a$ independent of $k_{x}$ and $k_{y}$, where $W$ defines the bandwidth. It is also convenient to consider the case of a half-filled band, i.e. $a=n_{\mathrm{e}}^{-1 / 3}$, where $n_{\mathrm{e}}$ is the electron density. We study this pedagogical toy model in order to illustrate the general relationship discussed earlier 9 between spiral magnetic order and spin supercurrents, not with the objective of modeling any specific material. In practice helimagnetism occurs for various reasons in several different types of materials; see for example Ref. 17 and work cited therein. We will briefly discuss some of these materials in Sections IV and V.

Choosing a quantization axis perpendicular to the spiral-order plane, the spin-projected current density is given by

$$
\mathbf{j}_{\sigma}=\frac{e}{V} \sum_{\mathbf{k}} \frac{\partial \epsilon_{\mathbf{k}}}{\partial(\hbar \mathbf{k})}\left\langle c_{\mathbf{k} \sigma}^{\dagger} c_{\mathbf{k} \sigma}\right\rangle
$$

The number operator average in the mean-field-theory state can be expressed in terms of Fermi occupation factors for the quasiparticles of the Hartree-Fock Hamiltonian leading to

$$
\mathbf{j}_{\uparrow}=\frac{e}{V} \sum_{\mathbf{k}} \frac{\partial \epsilon_{\mathbf{k}+\mathbf{Q} / 2}}{\partial(\hbar \mathbf{k})}\left[\sin ^{2} \Theta_{\mathbf{k}} f\left(E_{\mathbf{k}}^{-}\right)+\cos ^{2} \Theta_{\mathbf{k}} f\left(E_{\mathbf{k}}^{+}\right)\right] .
$$

It is then straightforward to show that $\epsilon_{\mathbf{k}}=\epsilon_{-\mathbf{k}}$ implies that $\mathbf{j}_{\downarrow}=-\mathbf{j}_{\uparrow}$.

An alternative expression follows from Eq. (1.2), which was discussed earlier ${ }^{9}$ and is explained in more detail in Appendix A The mean-field theory expression for the energy of a state with spiral-wavevector $\mathbf{Q}$ is

$$
\epsilon(\mathbf{Q})=\frac{h^{2}}{U}+\frac{1}{V} \sum_{\mathbf{k}}\left[E_{\mathbf{k}}^{+} f\left(E_{\mathbf{k}}^{+}\right)+E_{\mathbf{k}}^{-} f\left(E_{\mathbf{k}}^{-}\right)\right] .
$$

For all the numerical results discussed in this paper we explicitly checked the equivalence of Eqs. (1.2) and (2.7). Note that a spin current can be present even though the quasiparticle population is in equilibrium. Elastic scattering from occupied to unoccupied quasiparticle states cannot provide a spin-current decay mechanism. The spin currents are instead carried collectively, as in the case of dissipationless charge transport in BCS superconductors, and can decay only by collective processes 18

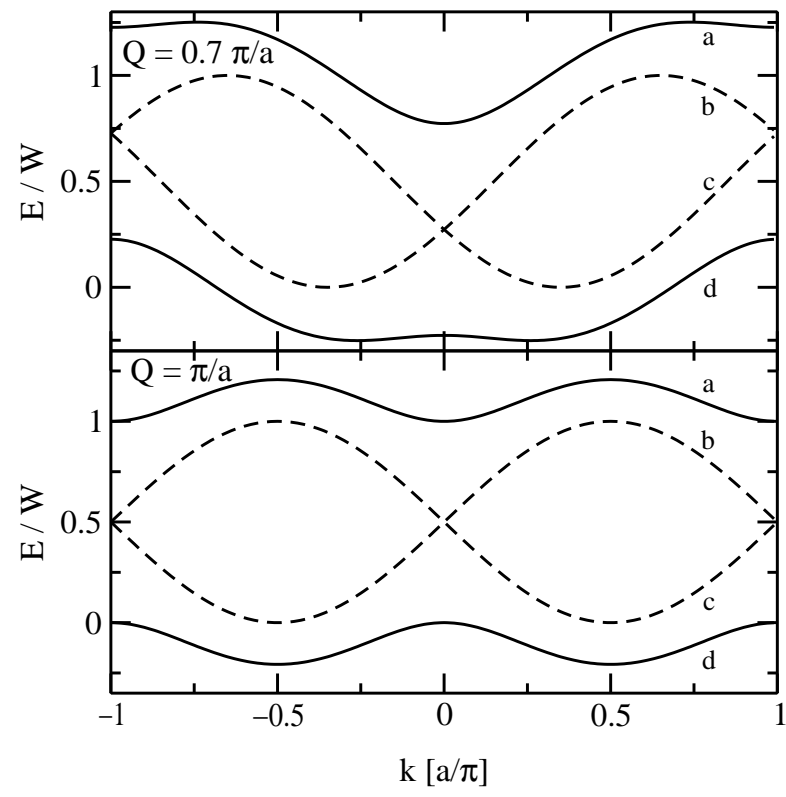

FIG. 2: Quasiparticle bands $E_{k_{z}}^{+}$(a) and $E_{k_{z}}^{-}$(d) for two different values of $Q$. The interaction strength $U$ is characterized by $2 n_{\mathrm{e}} U /(\pi W)=1$. For comparison, we also show the dispersion relations $\epsilon_{k_{z}+Q / 2}$ (b) and $\epsilon_{k_{z}-Q / 2}$ (c) for zero order parameter as dashed lines.

that allow the phase, which specifies the spiraling magnetization orientation, to slip.

\section{Numerical results}

Our numerical results are summarized in Figs. 2 and 3 . We choose $Q=Q_{z}$ to be in the $\hat{\mathbf{z}}$-direction. In Fig. 2 plot the quasiparticle energies $E_{\mathbf{k}}^{ \pm}$for $Q=0.7 \pi / a$ and $Q=\pi / a$.

The dashed curves represent $\epsilon_{k_{z} \pm Q / 2}$ for spin-up and spin-down electrons, respectively; note that the bands are degenerate for $k_{z} a=\pi$ when the order parameter vanishes. The quasiparticle bands $E_{k_{z}}^{ \pm}$(solid lines) are split by $\sqrt{\left(\epsilon_{\mathbf{k}+\mathbf{Q} / 2}-\epsilon_{\mathbf{k}-\mathbf{Q} / 2)^{2}+4 h^{2}}\right.}$, weakening the dispersion of both the occupied band and the empty band. The self-consistently determined values of the order parameter $s_{Q}$, the magnetic condensation-energy density $\epsilon_{\text {cond }}=\epsilon_{0}-\epsilon(Q)$ (where $\epsilon_{0}=n_{\mathrm{e}} W(1 / 2-1 / \pi)$ is the energy density of the state with $h=0$ ), and the spin supercurrent density $j=j_{\uparrow}=-j_{\downarrow}=$ are plotted as a function of the spiral wave vector $Q$ in Fig. 3] For the half-filled band case we consider the condensation energy is maximized for $Q=\pi / a$. In accord with Eq. (1.2) no spin supercurrent is present at this value of $Q$. For other values of $Q$ the derivative $\partial \epsilon(Q) / \partial Q$ is finite and the spin supercurrent is non-zero. In Fig. 3 we have used dashed lines in the regions where $\partial j(Q) / \partial Q$ is negative, to emphasize that the spiral state is not metastable. Energy can always be gained in this regime without changing the total number of phase windings by introducing domains 


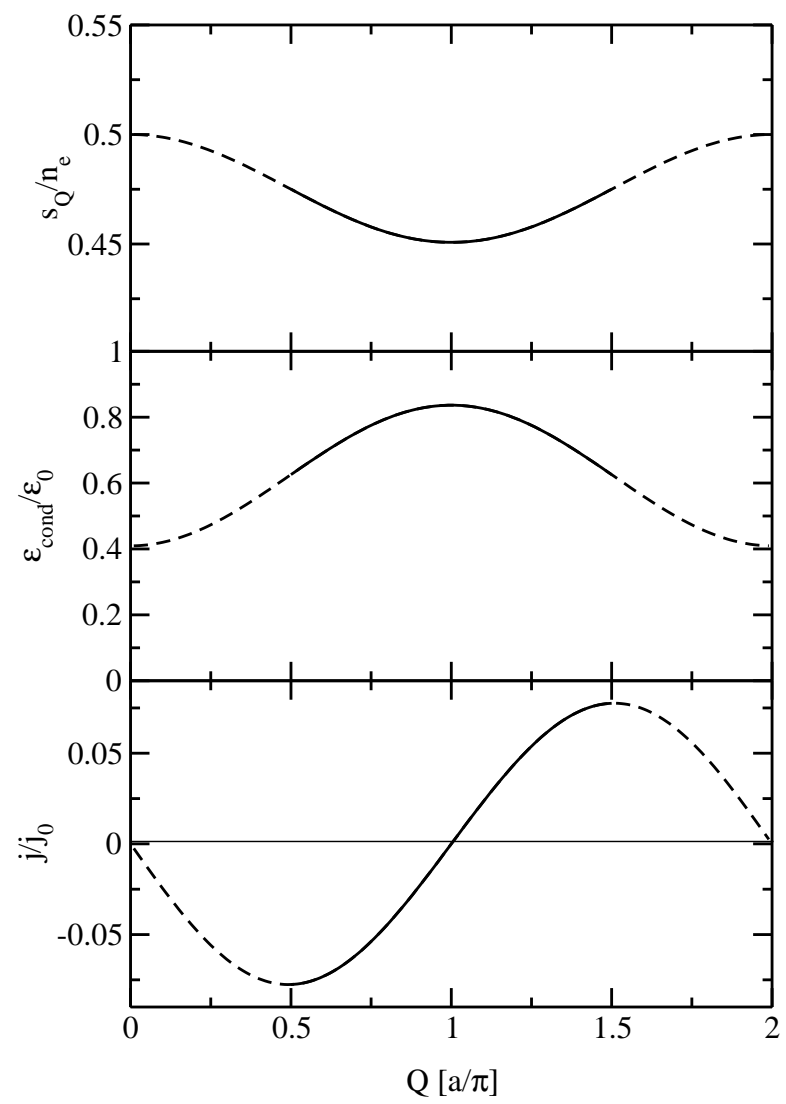

FIG. 3: The order parameter $s_{Q}$ normalized to the electron density $n_{\mathrm{e}}$, the magnetic condensation-energy density $\epsilon_{\text {cond }}$ normalized to the energy density $\epsilon_{0}$ of the disordered state, and the spin supercurrent density $j=j_{\uparrow}=-j_{\downarrow}$ normalized to $j_{0}=e n_{\mathrm{e}} v_{k_{F}}$ as a function of the ordering wave vector $Q$ for $2 n_{\mathrm{e}} U /(\pi W)=1$. The dashed lines indicate an instability regime against phase separation into regions with larger and smaller $Q$.

with different phase winding rates.

This example clearly shows that spiral magnetization configurations do not necessarily lead to spin supercurrents; instead they occur when the system is driven from its ground state into a state with a non-optimal spiral wavevector $Q$. In the case of a ferromagnet, i.e., a system for which the energy is minimized by $Q=0$, it might ${ }^{9}$ be possible to do this by driving the system from equilibrium using spin-selective transport currents supplied by ferromagnetic electrodes. This possibility has not yet been demonstrated experimentally, however, and may require combinations of material characteristics and geometries that are difficult to achieve. This difficulty motivates trying to find other strategies for realizing persistent spin currents. As we discuss in Section IV and $\mathrm{V}$ of this paper, in the case of a system with a spiral ground state persistent spin currents can be generated simply by applying an external magnetic field in the $\hat{\mathbf{x}}$ $\hat{\mathbf{y}}$ plane. The in-plane field drives the spiral state to a soliton lattice state which does carry a persistent spin current. Before turning to this central portion of our paper, we first briefly comment on the relationship between our discussion of persistent currents in ferromagnets and Anderson's discussion ${ }^{19}$ of superconductivity in terms of magnetic order in an effective spin model.

\section{ANALOGY WITH BCS THEORY}

To understand the physics behind our results for the dissipationless spin currents it is instructive to invert Anderson's analogy and address magnetic properties in terms of superconductivity. An analogy between Josephson junctions and tunnel junctions between ferromagnetic metals has been presented recently ${ }^{20}$ which is in the same spirit as the present discussion. The spin supercurrents discussed in this paper and the electrical supercurrents supported by a Cooper pair condensate in BCS superconductors appear in a similar way when a particlehole transformation is performed in one of the spin subspaces, say for spin down, to convert particle-particle order into particle-hole order. For example, the definition of the order parameter in the microscopic model, $s_{\mathbf{Q}}=(1 / V) \sum_{\mathbf{k}}\left\langle c_{\mathbf{k}+\mathbf{Q} / 2, \uparrow}^{\dagger} c_{\mathbf{k}-\mathbf{Q} / 2, \downarrow}\right\rangle$, is mapped to the order parameter of a superconductor with a momentum $\mathbf{Q}$ pair condensate by this transformation. A finite value of the order parameter $s_{\mathbf{Q}}$ corresponds to the formation of "Cooper pairs", consisting of an electron and a hole. These pairs carry no electric charge so that no charge supercurrent arises from the order. The formation of pairs with a finite momentum is partly analogous to the process leading to Fulde-Ferrell-Larkin-Ovchinnikov states 21 in a superconductor.

We can achieve a qualitative understanding of our numerical results for the persistent spin current by combining Eq. (1.2), Eq. (2.3) and Eq. (2.8), and ignoring the dependence of the order parameter magnitude on $\mathbf{Q}$. Then the contribution to the current from each wavevector $\mathbf{k}$ in Eq. 2.8 depends only on the velocities of the electron and the hole in the "Cooper pair", $\mathbf{v}_{\mathbf{k}}=\partial \epsilon_{\mathbf{k}} / \partial(\hbar \mathbf{k})$ and $\mathbf{v}_{-\mathbf{k}}=-\mathbf{v}_{\mathbf{k}}$. (These bare velocities can be identified with the slopes of the dashed lines in Fig. 2) At finite $\mathbf{Q}$, however, the velocity of a "Cooper pair" is finite and the sum over all wavevectors $\mathbf{k}$ in general produces a result that is not zero. Thinking in this way we can see directly that the point $Q=\pi / a$, for which we found in the previous section that the condensation energy is maximal and the total spin supercurrent is zero, is special. The reason is that the contribution of each "Cooper pair" is neutralized by that of another pair with reversed wave vectors for both electron and the hole.

\section{PERSISTENT SPIN CURRENTS IN HELIMAGNETS}

Two classes of helimagnets have been extensively studied in the past. The first is comprised of MnSi and related 
materials that have long-period helical ground states because of the Dzyaloshinskii ${ }^{22}$ instability of ferromagnetism in systems without inversion symmetry. These systems have been studied extensively very recently ${ }^{23}$ because they provide an example of an itinerant-electron magnetic system in which the ordering temperature can conveniently be driven to zero by applying pressure, and because they appear to show non-Fermi-liquid behavior associated with this nearby quantum critical point. They also have the potential advantage for the phenomenon of interest here that the chirality degeneracy, present in the microscopic model studied in Section II for example, is lifted 24 by broken inversion symmetry. In this case however, the plane of the spiral magnetic order is fixed only indirectly by anisotropy in the gradient term in the Landau-Ginzburg energy functional and the easy plane anisotropy that we require is rather weak.

The second class of materials consists of the heavy metals Tb, Dy, and Ho which have helical ground states due to frustration induced by RKKY interactions between the rare-earth moments and do have strong easy-plane anisotropy ${ }^{25,26,27}$ At present, it appears to us that these are the most promising materials for the realization of persistent spin currents.

In the case of a superconductor, charge supercurrents can be generated quite simply by biasing the sample with an external current source. In the case of an easy-plane ferromagnet, spin supercurrents can be generated by biasing the systems with an external spin current. One possible scheme for realizing such a bias using four ferromagnetic contacts is discussed in Ref. 9, but its success depends on avoiding spin-flip processes at the interfaces and on having thin-film samples with spin-diffusion lengths that are larger than the sample size. For systems with a spirally-ordered ground state, however, there is an easier and more straightforward way of generating persistent spin currents which does not depend on achieving spin-current biasing. Instead, persistent spin currents can be generated in the ground state of the system simply by applying a magnetic field in the plane defined by the spiral order parameter. In the continuum model that we study, the persistent-current state in a helimagnet is a soliton-lattice state for which the lattice Bloch wavevector is not a good quantum number. For this reason we cannot easily describe these states microscopically. We therefore use a phenomenological model for the following discussion.

\section{A. Magnetic order in the presence of an in-plane magnetic field}

A magnetic field in the $\hat{\mathbf{x}}-\hat{\mathbf{y}}$ plane alters the spiral state. At sufficiently large field strengths, it is clear that the magnetic field will lead to nearly uniform spinpolarization along the field direction. As we show, for fields below a critical strength, the spiral state is driven by the field to a soliton-lattice state, in which the mag- netization orientation slips periodically from the fielddirection orientation.

We assume that due to easy-plane anisotropy the magnetization is in the $\hat{\mathbf{x}}-\hat{\mathbf{y}}$ plane and has a spatially constant amplitude. The orientation variation of the magnetization density $\mathbf{m}(\mathbf{r})=g \mu_{\mathrm{B}}\langle\mathbf{s}(\mathbf{r})\rangle$ is parametrized by the angular variable $\vartheta(\mathbf{r})$ via $\mathbf{m}(\mathbf{r})=m_{0}[\cos (\vartheta(\mathbf{r})) \hat{\mathbf{x}}-$ $\sin (\vartheta(\mathbf{r})) \hat{\mathbf{y}}]$. For clarity, we choose $\mathbf{Q}^{\star}$ in $\hat{\mathbf{z}}$-direction, $\mathbf{Q}^{\star}=\left(0,0, Q^{\star}\right)$ so that $\vartheta$ only depends on the $\hat{\mathbf{z}}$-position. However, the following discussion does not depend on this specific choice. To determine the magnetic order at $T=0$ we have to minimize the energy density

$$
\epsilon=\int \frac{d z}{L_{z}}\left[\frac{\rho_{\mathrm{s}}}{2}\left|\partial_{z} \vartheta(z)-Q^{\star}\right|^{2}-\mathbf{B} \cdot \mathbf{m}(z)\right],
$$

where $L_{z}$ is the length of the sample in $\hat{\mathbf{z}}$-direction, the spin stiffness $\rho_{\mathrm{s}}$ characterizes the energy cost for spiral order with wavectors close to the minimal value $\mathbf{Q}^{\star}$, and $\mathbf{B}$ is the in-plane magnetic field.

Without loss of generality we choose the inplane magnetic field along the $\hat{\mathbf{x}}$ direction, $\mathbf{B}=(B, 0,0)$. For this particular choice of directions, $\vartheta$ is the angle between the local magnetization direction and the external magnetic field. This model for a spiral state in an external field (Eq. (4.1)) is equivalent to the Pokrovsky-Talapov (PT) model, reviewed for example by $\mathrm{P}$. Bak,$\stackrel{28}{r}$ used originally to model commensurate-incommensurate transitions and more recently to model the influence of an in-plane field on spontaneous coherence broken symmetry states in bilayer quantum Hall systems. In using this model we assume that the energy density depends on the gradient of the orientation angle $\vartheta$ and that, for qualitative purposes, we can expand around the value of the gradient that minimizes the energy density. This chiral model favors a particular sign for $\partial_{z} \vartheta$ and does not account for the symmetry-based expectation that the energy density will have minima for $\partial_{z} \vartheta= \pm Q^{*}$. Hence it accounts only for the effect of a magnetic field on a spiral state domain with a particular chirality; we speculate later on persistent spin currents near boundaries between domains with opposite chirality. A more general model of the gradient energy that does not favor a particular chirality would have the form $\int\left(d z / L_{z}\right)\left(\rho_{\mathrm{s}} / 2\right)\left[-\left(\partial_{z} \vartheta\right)^{2} / 2+\left(\partial_{z} \vartheta\right)^{4} / 4 Q^{* 2}\right]$ plus a constant. Finally, our continuum model ignores the possibility of locking to configurations that are commensurate with the underlying lattice. Locking to commensurate configurations has been studied for helimagnets in fields for both non-chiral ${ }^{29}$ and chiral ${ }^{30}$ models. Indeed, magnetoelastic coupling is known to play an important and complex role ${ }^{27}$ in the response of helimagnets to external magnetic fields. Our use of a chiral continuum model for the following discussion certainly does not capture the full richness of real helimagnets in external magnetic fields. Our intention here is to demonstrate simply the general property that external fields induce persistent spin currents within each chiral domain, using a model that is well understood 28,31 . In the following discussion we use well known properties of the classical 


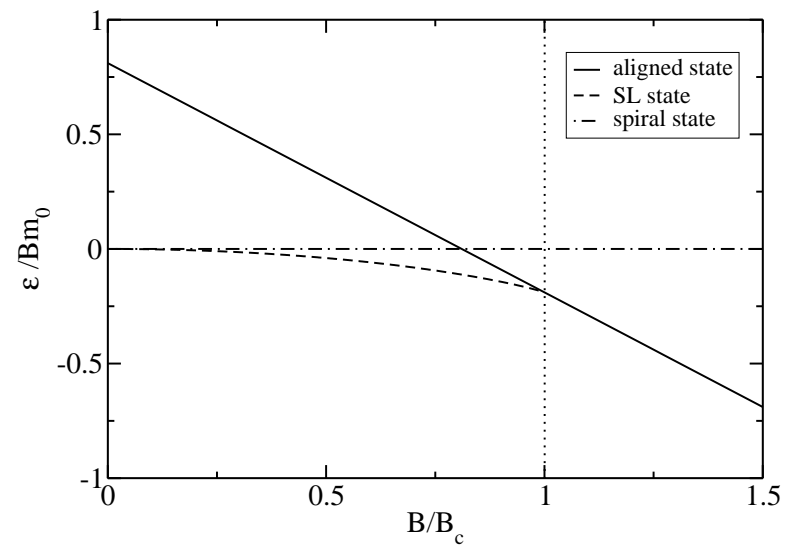

FIG. 4: Energy versus in-plane magnetic field for our spiral state model. The soliton-lattice state minimizes the model energy for $B<B_{c}$, while the state in which the magnetization is aligned with field minimizes the energy for $B>B_{c}$. The soliton lattice state energy approaches the spiral state energy as $B \rightarrow 0$. The energies are normalized to $B_{c} m_{0}$.

sine-Gordon model to evaluate the persistent spin current within a chiral domain of a helimagnet.

Minimization of the energy with respect to variations in $\vartheta(z)$ leads to the sine-Gordon equation

$$
\frac{\partial^{2} \vartheta(z)}{\partial z^{2}}=\frac{1}{\xi^{2}} \sin (\vartheta(z))
$$

where $\xi=\sqrt{\rho_{\mathrm{s}} / B m_{0}}$. For fields $B$ larger than a critical value $B_{c}$, the ground state magnetization is uniform with $\vartheta(z) \equiv 0$. For $B$ just below $B_{c}$ the energy can be lowered by incorporating isolated $2 \pi$ solitons; $\vartheta_{\mathrm{ss}}(z)=4 \arctan \left[\exp \left( \pm\left(z-z_{0}\right) / \xi\right)\right]$ for a soliton centered at $z_{0}$. At $B_{c}$, the uniform state has the same energy as a state which accommodates a single soliton. By comparing energies we find that in terms of the notation defined above

$$
B_{c}=\frac{\pi^{2}}{16} \frac{\rho_{\mathrm{s}}}{m_{0}} Q^{\star 2} .
$$

For $B<B_{c}$ the magnetic state can be described as a soliton lattice (SL) with period $a$. The associated wave vector $Q=2 \pi / a$ varies from $Q=0$ at $B=B_{c}$ to $Q=Q^{\star}$ at $B=0$. In the latter limit the soliton-lattice state approaches the spiral magnetic order state, discussed from a microscopic point of view in the first part of the paper. The total phase change along the sample is given by $Q L_{z}$.

The results collected in the following paragraph are adapted from Ref. 31 and earlier work cited therein. Minimization of the model's energy in the soliton lattice state gives, $\cos (\vartheta(z) / 2)=-\operatorname{sn}\left[\left(z-z_{0}\right) / \eta \xi, \eta\right]$ where sn denotes the sine-amplitude Jacobian elliptic function, 32 and $\eta$ is a constant which depends on the strength of the magnetic field $B$;

$$
\frac{B}{B_{c}}=\left(\frac{\eta}{E(\eta)}\right)^{2}
$$

where $E(\eta) \equiv \int_{0}^{\pi / 2} d \beta \sqrt{1-\eta^{2} \sin ^{2} \beta}$ denotes the complete elliptic integral of the second kind 32 From that we see that the limit $\eta=1$ applies at the SL state with $B=B_{c}$, and that $\eta=0$ describes the opposite situation of a vanishing in-plane magnetic field $B=0$. The wave vector $Q$ of the $\mathrm{SL}$ is given by

$$
\frac{Q}{Q^{\star}}=\frac{(\pi / 2)^{2}}{K(\eta) E(\eta)},
$$

where $K(\eta) \equiv \int_{0}^{\pi / 2} d \beta / \sqrt{1-\eta^{2} \sin ^{2} \beta}$ denotes the complete elliptic integral of the first kind ${ }^{32}$ We note that $Q=0$ for $B=B_{c}$, and $Q=Q^{\star}$ for $B=0$. Finally, the expression for the energy density of the SL, which we relate to persistent spin currents below, is

$$
\epsilon=\frac{\rho_{\mathrm{s}}}{2} Q^{\star 2}-B m_{0}\left(\frac{2}{\eta^{2}}-1\right) \text {. }
$$

The SL energy density is plotted as a dashed line in Fig. (4). For $B<B_{c}$ the SL is energetically favored over a uniform state, whose energy is $(1 / 2) \rho_{\mathrm{s}} Q^{\star 2}-B m_{0}$. For $B \neq 0$ the SL lattice is always favored over the spiral state (with wave vector $Q^{\star}$ ). The energy of the latter state is the zero of energy of our model.

A cautionary note is appropriate at this point. We do not expect that the expansion of the gradient energy around the spiral wavevector that appears in our model will be realistic for typical spiral magnets all the way down to the $Q=0$ state that is favored by the in-plane field. It follows that our model is most realistic physically in the $B<B_{c}$ region.

\section{B. Spin supercurrent}

We now demonstrate how the in-plane magnetic field gives rise to a persistent spin current. To derive an expression for the spin current density we introduce a spin-dependent, spatially constant vector potential $\mathbf{A}_{\sigma}=$ $A_{\sigma} \hat{\mathbf{z}}$. The energy density of the system then reads

$$
\begin{aligned}
\epsilon=\frac{1}{2} \sum_{\sigma} \int & \frac{d z}{L_{z}}\left[\frac{\rho_{\mathrm{s}}}{2}\left(\frac{\partial \vartheta}{\partial z}-Q^{\star}+\sigma \frac{2 e}{\hbar c} A_{\sigma}\right)^{2}\right. \\
& \left.-B m_{0} \cos \left(\vartheta+\frac{e\left(A_{\uparrow}-A_{\downarrow}\right)}{\hbar c} z\right)\right] .
\end{aligned}
$$

Note that here we have explicitly used the coincidence of $\vartheta$ with the angle between the magnetization direction and the magnetic field; for a different orientation of the magnetic field in the $\hat{\mathbf{x}}-\hat{\mathbf{y}}$ plane, Eq. (4.7) adopts a different form. The spin-dependent current density follows from 


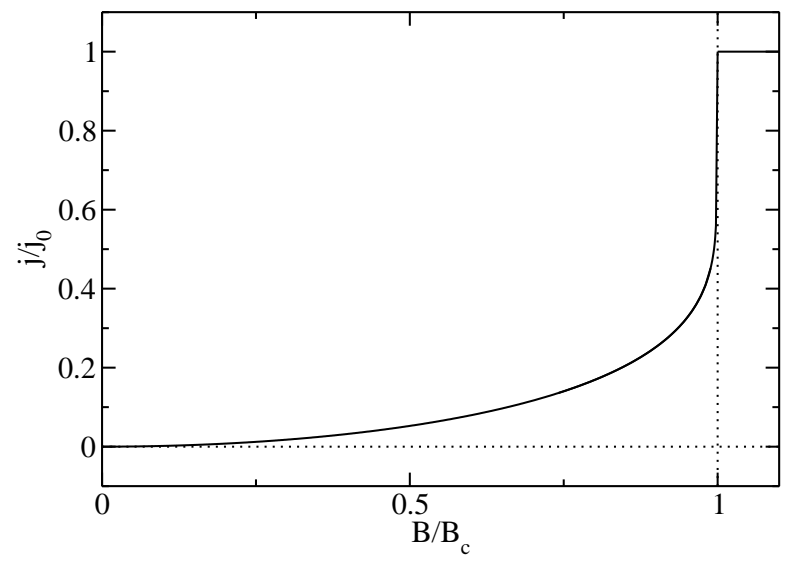

FIG. 5: Spin supercurrent density $j \equiv j_{\uparrow}$ induced by an inplane magnetic field. In the spirally ordered state at $B=0$ there is no current and for $B>B_{c}$ the current is constant.

the derivative $j_{\uparrow}=c \partial \epsilon\left(A_{\uparrow}, A_{\downarrow}\right) / \partial A_{\uparrow}$ at $A_{\uparrow}=A_{\downarrow}=0$. Making use of the relation $\int d z \sin (\vartheta(z))=0$, that holds for all soliton lattices with arbitrary wave vector, we find that

$$
j_{\uparrow}=\frac{e}{\hbar} \rho_{\mathrm{s}}\left(Q-Q^{\star}\right)=-j_{\downarrow} .
$$

At $B=0$ we have a spiral state with $Q=Q^{\star}$, and the spin current vanishes. With increasing strength of the $B$ field the wave vector $Q$ decreases and a finite spin supercurrent arises. At $B=B_{c}$ the spin current $j_{\uparrow}$ reaches its maximal value $j_{0} \equiv-(e / \hbar) \rho_{\mathrm{s}} Q^{\star}$. The limit of small magnetic fields, $B \ll B_{c}$, is described by

$$
\frac{j_{\uparrow}}{j_{0}} \approx \frac{\pi^{4}}{512}\left(\frac{B}{B_{c}}\right)^{2}
$$

The spin current density as a function of the in-plane magnetic field is shown in Fig. 5

In conclusion, the SL state of a helimagnet carries a finite persistent spin current, without any electrical current, when a magnetic field is applied in the plane defined by the spiral order.

\section{DISCUSSION}

We have shown in this paper that persistent spin currents can be induced in helimagnets by applying a magnetic field in the plane defined by the spiral order. It is interesting to speculate on the possibility of exploiting this effect for new types of spintronic devices. Current spintronic devices make use of magnetotransport effects like anisotropic magnetoresistance, giant magnetoresistance, ${ }^{33}$ and tunnel magnetoresistance,, 34 all of which follow from the dependence of quasiparticle transport on the collective magnetic state. The effect we have discussed here is distinctly different in that it

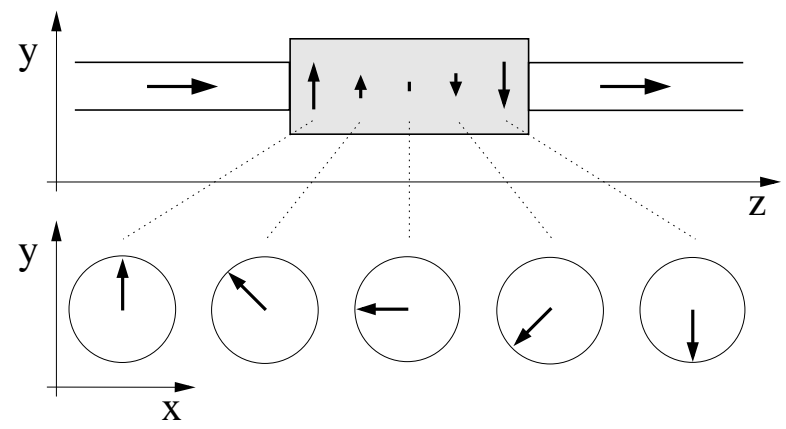

FIG. 6: Sketch of the setup discussed in the text. The direction of the magnetization is indicated by the arrows. In the wires it is parallel to the $\hat{\mathbf{z}}$-axes along the transport direction, while in the helimagnet (shaded grey) it is circulating in the $\hat{\mathbf{x}}-\hat{\mathbf{y}}$ plane as shown in the lower part of the sketch.

is an equilibrium spin current that is carried collectively rather than by quasiparticles.

To illustrate the possibility of realizing interesting magnetotransport effects based on field-induced persistent currents in helimagnets, consider the case illustrated in Fig. 6] We consider current flow along a ferromagnetic wire which contains a helimagnet. The magnetization direction in the ferromagnets is assumed to be aligned along the direction of the wire by magnetostatic energy, and the electrical current in the ferromagnet will be spinpolarized along the direction of the magnetization. We also assume that the helimagnet is grown so that it has its wavevector along the wire direction and the spiral magnetization is in the perpendicular plane; this would be the case for example for rare earth metal single-crystal helimagnets with their hcp c-axis aligned with the wire. The quasiparticles of the helimagnet would then tend to have their spins aligned in the plane perpendicular to the wire axis, suppressing their ability to carry currents that are spin-polarized along the wire axis, and increasing the resistance of the overall system. In the presence of a field perpendicular to the wire axis, however, the helimagnet would have a persistent spin current polarized along the wire axis, eliminating the need to carry the spin current with quasiparticles. We predict that because of the persistent spin currents, an in-plane field could therefore alter the resistance of this system. Since the persistent spin current has a sign that depends on the chirality of the spiral, the sign of the resistance depends on this quantity. The effect should be strongest in samples with a single chiral domain.

This same effect could also be of interest in a similar setup where the helimagnet is connected to normal metallic leads. Then the spin current in the helimagnet results in a different resistance for the electron spins entering from the leads. In this fashion the helimagnet could act as a tunable spin filter which is controlled by an external magnetic field.

It may also be possible to realize spin torque ${ }^{35}$ effects in helimagnets that are distinct from those which occur 
in ferromagnets. Spin torque occurs when the spin polarization of a quasiparticle current changes with position by altering the spin distribution of current-carrying states, transferring the spin carried by the quasiparticle to the collective magnetic coordinate. The field-induced persistent spin current in a helimagnet will have a strong spatial dependence, a sign change in fact, near a boundary surface between domains with opposite chirality. The divergence of this spin current makes a contribution to the time-dependent spin polarization that is cancelled by the torque due to the external magnetic field when the domain wall is in equilibrium. When the domain wall is not in equilibrium, for example by being displaced from a pinning center, these two contributions will not cancel and the net torque will lead to a time-dependent magnetization orientation. Conversely, the equilibrium position of a pinned domain wall will be sensitive to the presence of persistent currents and will therefore be altered by an in-plane field.

In conclusion, we have studied the relation between spiral magnetic order and dissipationless spin transport. We have demonstrated the possibility of dissipationless spin currents in states with equilibrium quasiparticle populations. These spin currents are always associated with spiral magnetic order. On the other hand, the existence of spiral magnetic order is, in general, not sufficient to guarantee collective spin transport. In particular helimagnets, which have spiral ground states, do not support persistent spin currents. However, persistent spin currents can be induced in these systems simply by applying an external magnetic field.

We acknowledge helpful discussions with Martin Bønsager, Herbert Capellmann, Jan Martinek, Herbert
Schoeller, Michael Steinitz and Leo Radzihovsky. The work is part of the CFN which is supported by the DFG. We acknowledge funding by the European Training Network RTN2-2001-00440 and by the DFG-Emmy-Noetherprogram KO1987/2. Work at the University of Texas was supported by the Welch Foundation and by the DOE under grant DE-FG03-02ER45958.

\section{APPENDIX A: PROOF OF EQ. (2)}

In order to prove that the spin-current density is related to the derivative of the total energy with respect to the wave vector $\mathbf{Q}$ as given in Eq. (1.2) we extend the Hartree-Fock Hamiltonian Eq. (2.1) by formally introducing an additional parameter $\tilde{\mathbf{Q}}$ via the replacement $\epsilon_{\mathbf{k}+\mathbf{Q} / 2} \rightarrow \epsilon_{\mathbf{k}+(\mathbf{Q}+\tilde{\mathbf{Q}}) / 2}$ and $\epsilon_{\mathbf{k}-\mathbf{Q} / 2} \rightarrow$ $\epsilon_{\mathbf{k}-(\mathbf{Q}+\tilde{\mathbf{Q}}) / 2}$. Our original Hamiltonian Eq. (2.1) corresponds to $\left.\mathcal{H}^{\mathrm{HF}}(\mathbf{Q}, \tilde{\mathbf{Q}})\right|_{\tilde{\mathbf{Q}}=0}$. The quasiparticle ground state for given $\mathbf{Q}$ and $\tilde{\mathbf{Q}}$ is an eigenstate and, therefore, the Hellmann-Feynman theorem applies,

$$
\left.\frac{\partial\left\langle\mathcal{H}^{\mathrm{MF}}(\mathbf{Q}, \tilde{\mathbf{Q}})\right\rangle}{\partial \tilde{\mathbf{Q}}}\right|_{\tilde{\mathbf{Q}}=0}=\left.\left\langle\frac{\partial \mathcal{H}^{\mathrm{MF}}(\mathbf{Q}, \tilde{\mathbf{Q}})}{\partial \tilde{\mathbf{Q}}}\right\rangle\right|_{\tilde{\mathbf{Q}}=0}
$$

By comparing the r.h.s with Eq. (2.6) we find that Eq. A1 equals to $V(\hbar / 2 e)\left(\mathbf{j}_{\uparrow}-\mathbf{j}_{\downarrow}\right)$. To evaluate the l.h.s of Eq. A1 we observe that the quasiparticle energies $E_{\mathbf{k}}^{ \pm}$for $\mathcal{H}^{\mathrm{MF}}(\mathbf{Q}, \tilde{\mathbf{Q}})$ as well as the value of $h$ depend on $\mathbf{Q}$ and $\tilde{\mathbf{Q}}$ only in the combination $\mathbf{Q}+\tilde{\mathbf{Q}}$. As a consequence, we can replace $\partial / \partial \tilde{\mathbf{Q}}$ by $\partial / \partial \mathbf{Q}$. Together with $\mathbf{j}_{\uparrow}=-\mathbf{j}_{\downarrow}$, this immediately proves Eq. (1.2).
1 J. Bardeen, L.N. Cooper, and J.R. Schrieffer, Phys. Rev. 108, 1175 (1957).

2 X.-G. Wen and A. Zee, Phys. Rev. Lett. 69, 1811 (1992); K. Yang et al., Phys. Rev. Lett. 72, 732 (1994); K. Moon et al., Phys. Rev. B 51, 5138 (1995).

3 J. Kyriakidis, D. Loss, and A.H. MacDonald, Phys. Rev. Lett. 83, 1411 (1999); 85, 2222 (2000); J. Kyriakidis and L. Radzihovsky, Phys. Rev. B 64, 201314 (2001).

${ }^{4}$ I.B. Spielman, J.P. Eisenstein, L.N. Pfeiffer, and K.W. West, Phys. Rev. Lett. 84, 5808 (2000); Phys. Rev. Lett. 87, 036803 (2001); M. Kellogg, I.B. Spielman, J.P. Eisenstein, L.N. Pfeiffer, and K.W. West Phys. Rev. Lett. 88, 126804 (2002).

${ }^{5}$ L. Balents, L. Radzihovsky, Phys. Rev. Lett. 86, 1825 (2001); A. Stern et al., ibid. 1829 (2001); M. Fogler and F. Wilczek, ibid. 1833 (2001).

6 Yu.E. Lozovik and V.I. Yudson, Zh. Eksp. Teor. Fiz. 71, 738 (1976) [Sov. Phys. JETP 44, 389 (1976)]; S.I. Shevchenko, Sov. J. Low. Temp. Phys. 2, 251 (1976).

7 L.V. Keldysh and Yu.V. Kopaev, Sov. Phys. Solid State 6, 2219 (1965); D. Jérome, T.M. Rice, and W. Kohn, Phys. Rev. 158, 462 (1967); B.I. Halperin and T.M. Rice, Rev. Mod. Phys. 40, 755 (1968); R.R. Guseinov and L.V.
Keldysh, Zh. Eksp. Teor. Fiz. 63, 2255 (1972) [Sov. Phys. JETP 36, 1193 (1973)].

8 L.V. Butov, A.C. Gossard, and D.S. Chemla, Nature 418, 751 (2002); D. Snoke, S. Denev, Y. Liu, L. Pfeiffer, and K. West, Nature 418, 754 (2002).

9 J. König, M.C. Bønsager, and A.H. MacDonald, Phys. Rev. Lett. 87, 187202 (2001).

10 D. Loss, P. Goldbart, and A.V. Balatsky, Phys. Rev. Lett. 65, 1655 (1990).

11 X.-C. Gao and T.-Z. Qian, Phys. Rev. B 47, 7128 (1993).

12 F. Schütz, M. Kollar, and P. Kopietz, Phys. Rev. Lett. 91, 017205 (2003).

13 Equation (1.2) is partly related to the connection between the exchange coupling between ferromagnets separated by a tunnel junction and the spin currents that flow between them that has been discussed in J.C. Slonczewski, Phys. Rev. B 39, 6995 (1989).

14 H. Capellmann, Phys. Stat. Sol. (b) 64, K143 (1974).

15 A. Auerbach, Interacting Electrons and Quantum Magnetism (Springer, New York, 1994).

16 E.C. Stoner, Proc. Roy. Soc. A154, 656 (1936).

17 H. Kawamura, J. Phys.: Condens. Matter 10, 4707 (1998).

18 J.S. Langer and V. Ambegaokar, Phys. Rev. 164, 498 
(1967); D.E. McCumber, Phys. Rev. 172, 427 (1968); D.E. McCumber and B.I. Halperin, Phys. Rev. B 1, 1054 (1970); J. Kyriakidis and L. Radzihovsky, Phys. Rev. B 64, 201314 (2001).

19 P.W. Anderson, Phys. Rev. 112, 1900 (1958).

${ }^{20}$ F.S. Nogueira and K.-H. Bennemann, cond-mat/0302528

21 P. Fulde and R.A. Ferrell, Phys. Rev. 135, A550 (1964); A.I. Larkin and Yu. N. Ovchinnikov, Sov. Phys. JETP 20, 762 (1965). For recent work see for example K. Yang and D. F. Agterberg, Phys. Rev.Lett. 84, 4970 (2000).

22 I.E. Dzyaloshinskii, J.E.T.P 46, 1420 (1964) [Sov. Phys.JETP 19, 960 (1964).]

23 F.P. Mena, D. van der Marel, A. Damascelli, M. Fäth, A. A. Menovsky, and J. A. Mydosh, Phys. Rev. B 67, 241101 (2003); T. Vojta and R. Sknepnek Phys. Rev. B 64, 052404 (2001); C. Pfleiderer, S.R. Julian, and G.G. Lonzarich, Nature 414, 427 (2001).

24 P. Bak and J. H. Jensen, J. Phys. C 13, L881 (1980).

25 R.J. Elliot, Magnetic Properties of Rare Earth Metals (Plenum, London, 1972).

${ }^{26}$ M. O. Steinitz, M. Kahrizi, and D.A. Tindall, Phys. Rev. B 36, 783 (1987); N. Ali, F. Willis, M.O. Steinitz, M. Kahrizi, and D.A. Tindall, Phys. Rev. B 40, 11414 (1989).

27 M.L. Plumer, J. Appl. Phys. 67, 5280 (1990); Phys. Rev. B 44, 12376 (1991).

28 P. Bak, Rep. Prog. Phys. 45, 587 (1982).

29 A.B. Harris, E. Rastelli and A. Tassi, Phys. Rev. B 44,
2624 (1991).

30 C.S.O. Yokoi, L.H. Tang, and W. Chou, Phys. Rev. B 37, 2173 (1988).

31 See for example C.B. Hanna, A.H. MacDonald, and S.M. Girvin, Phys. Rev. B 63, 125305 (2001).

32 Table of Integrals, Series, and Products, edited by I. S. Gradshteyn and I. M. Rhyzik (Academic Press, New York, 2000), 6th ed.

33 M.N. Baibich,J. M. Broto, A. Fert, F. Nguyen Van Dau, F. Petroff, P. Etienne, G. Creuzet, A. Friederich, and J. Chazelas, Phys. Rev. Lett. 61, 2472 (1988); G. Binasch, P. Grünberg, F. Saurenbach, and W. Zinn, Phys. Rev. B 39, 4828 (1989).

34 M. Julliere, Phys. Lett. A 54, 225 (1975); J.S. Moodera, L.R. Kinder, T.M. Wong, and R. Meservey, Phys. Rev. Lett. 74, 3273 (1995).

35 J.C. Slonczewski, J. Magn. Magn. Mater. 159, L1 (1996); L. Berger, Phys. Rev. B 54, 9353 (1996); L. Berger, J. Appl. Phys. 81, 4880 (1997); J.A. Katine, F.J. Albert, and R.A. Buhrman, Appl. Phys. Lett. 76, 354 (2000); J.A. Katine, F.J. Albert, R.A. Buhrman, E.B. Myers, and D.C.Ralph, Phys. Rev. Lett. 84, 3149 (2000); E.B. Myers, D.C.Ralph, J.A. Katine, F.J. Albert, and R.A. Buhrman, J. Appl. Phys. 87, 5502 (2000); W.H. Rippard and R.A. Buhrman, J. Appl. Phys. 87, 6490 (2000). 